\title{
Relações entre contexto familiar e uso de drogas em adolescentes de ensino médio
}

\author{
Jairo Jose Garcia ${ }^{1}$ \\ Sandra Cristina Pillon² \\ Manoel Antônio dos Santos ${ }^{3}$
}

\begin{abstract}
Este estudo teve por objetivo descrever características do contexto familiar de adolescentes de ensino secundário e suas relações com o uso de substâncias psicoativas. Utilizou-se desenho de estudo descritivo transversal, do tipo survey, com amostra de 657 estudantes de ensino secundário da cidade de Leon, Nicarágua, correspondendo a $31 \%$ do universo investigado. As informações foram coletadas por meio de questionário autoadministrado e anônimo. Os resultados mostraram que 56\% dos adolescentes conviviam com ambos os pais e $32 \%$ somente com a mãe; $86 \%$ tinham boas relações com a mãe, porém $24 \%$ não mostravam confiança na figura materna. Em relação ao uso de substâncias psicoativas no núcleo familiar, $52 \%$ das famílias de origem dos/das adolescentes apresentavam antecedentes, sendo o pai o que normalmente fazia uso (42\%). Os resultados trazem contribuições relevantes para a elaboração de políticas públicas na área da saúde e o delineamento de estratégias de prevenção entre adolescentes.
\end{abstract}

Descritores: Adolescente; Transtornos Relacionados ao Uso de Substâncias; Drogas Ilícitas; Saúde Pública; Promoção da Saúde. \footnotetext{
Paulo, SP, Brasil. E-mail: masantos@ffclrp.usp.br.

Correspondencia:

Manoel Antônio dos Santos

Universidade de São Paulo

Faculdade de Filosofia, Ciências e Letras de Ribeirão Preto

Av. dos Bandeirantes, 3900

Bairro: Monte Alegre

CEP: 14040-902 Ribeirão Preto, SP, Brasil

E-mail: masantos@ffclrp.usp.br
}

${ }^{1}$ Médico, ciudad de Leon, Nicarágua. E-mail: jaigarci10@hotmail.com.

${ }^{2}$ Enfermeira, Doutora en Enfermagem. Profesor Asociado, Escola de Enfermagem de Ribeirão Preto, Universidade de São Paulo, Centro Colaborador da OMS para o Desenvolvimento da Pesquisa em Enfermagem, SP, Brasil. E-mail: pillon@eerp.usp.br

${ }^{3}$ Psicólogo, Doutor en Psicología. Profesor Doctor, Faculdade de Filosofia, Ciências e Letras de Ribeirão Preto, Universidade de São 


\section{Relaciones entre la situación familiar y el uso de drogas en adolescentes de la enseñanza secundaria}

Este estudio tuvo como objetivo describir características del contexto familiar de adolescentes de la enseñanza secundaria y sus relaciones con el uso de sustancias psicoactivas. Se utilizo un diseño de estudio descriptivo transversal, de tipo encuesta, con una muestra de 657 estudiantes de escuelas secundarias de la ciudad de León, en Nicaragua, correspondiendo a $31 \%$ del universo. La información fue recolectada a través de un cuestionario auto aplicado y anónimo. El 56\% del total convive con ambos padres y el $32 \%$ sólo con la madre. Tienen buenas relaciones con la madre pero no confían en ella, con $86 \%$ y $24 \%$ respectivamente. En relación al uso de sustancias en el núcleo familiar, el $52 \%$ de las familias de donde proceden las/los adolescentes tienen antecedentes de uso, siendo el padre el que generalmente usa $(42 \%)$. Los resultados aportan contribuciones relevantes para la elaboración de políticas públicas en salud y para el delineamiento de estrategias de prevención con adolescentes.

Descriptores: Adolescente; Trastornos Relacionados con Sustancias; Drogas Ilícitas; Salud Pública; Promoción de la Salud.

\section{Relations Between Family Context and Substance Abuse in High School Adolescents}

This study aimed to describe the family context characteristics of high school adolescents and their relation to psychoactive substance abuse. It is a cross-sectional and descriptive study, with a population of 657 secondary-school students in the city of Leon, Nicaragua, which corresponded to $31 \%$ of the research context. Data was collected through an anonymous questionnaire. Results show that $56 \%$ of the adolescents were living with both parents and $32 \%$ only with the mother, $86 \%$ declared a good relationship with the mother, but $24 \%$ did not show confidence in the mother figure. Regarding psychoactive substance use within the family context, $52 \%$ of the adolescents' family presented previous use events and, in $42 \%$ of cases, the father was the user. Results offer important contributions to design public healthcare policies and to outline prevention strategies for use among adolescents.

Descriptors: Adolescent; Substance-Related Disorders; Street Drugs; Public Health; Health Promotion.

\section{Introdução}

O fenômeno do uso de substâncias psicoativas por adolescentes constitui problema de saúde pública contemporâneo que vem despertando intensa preocupação no cenário internacional(1). O uso de drogas, lícitas ou ilícitas, constitui realidade palpável que penetrou os interstícios de praticamente todos os países $^{(2)}$. Todavia, ainda são escassas as investigações em algumas partes do mundo, como nos países da América Central, conforme mostram os estudos publicados neste periódico, nos últimos anos(1,3-5).

Nicarágua não está isolada em relação ao avanço das drogas, pois é parte do conglomerado que conforma as nações do mundo e, portanto, também está imersa no problema(6). Por outro lado, a globalização que, direta ou indiretamente, envolve tanto os países em desenvolvimento como os desenvolvidos, tem favorecido, em grande medida, que as drogas naturais ou sintéticas cheguem com extrema facilidade às mãos dos adolescentes que, por suas características de imaturidade e vulnerabilidade psicossocial, se tornam 
presas fáceis do tráfico. A abordagem do fenômeno das drogas em adolescentes da América Latina reveste-se de grande importância(7), já que, como é bem conhecido, em países como Nicarágua, o segmento formado por adolescentes e jovens corresponde a quase $65 \%$ da população total.

Os conhecimentos acerca da etiologia da farmacodependência na adolescência, e no ser humano em geral, têm sido ampliados nas últimas décadas, sendo focalizados sob múltiplos e diferentes pontos de vista. Como ocorre em todas as condições crônicas, os transtornos relacionados ao abuso de substâncias constituem entidade clínica multideterminada, na qual se pode distinguir elementos biológicos, psicológicos, ambientais, familiares e socioculturais. Esses componentes, por sua vez, podem ser articulados em uma perspectiva ecossistêmica(6).

Investigações sobre o fenômeno das drogas na adolescência revelam que, geralmente, é nessa etapa do ciclo vital que a maioria dos consumidores têm seu primeiro contato com as drogas ${ }^{(1,4-5,8-9)}$. Por essa razão, esse grupo etário é considerado prioritário para a investigação científica, assim como para a elaboração de estratégias de intervenção e programas de prevenção ao consumo de drogas.

Estudos de caracterização dos adolescentes, em relação ao consumo das drogas, tanto lícitas como ilícitas, são importantes para preencher um vazio de informações acerca dessa realidade na Nicarágua e contribuem para oferecer subsídios e insumos para a elaboração de políticas públicas, com vistas a prevenir o uso e abuso de substâncias ${ }^{(10)}$. A formulação de políticas públicas certamente ajudaria na diminuição dos índices de criminalidade e violência, tanto intrafamiliar como comunitária, que cercam o fenômeno das drogas, contribuindo para a redução do número de jovens mortos que tem crescido nos países latino-americanos, sendo adolescentes e jovens o grupo etário mais $\operatorname{afetado}^{(4-5,7)}$.

Em estudo realizado na cidade de León, Nicarágua, em 2000, foram entrevistados 150 jovens do sexo masculino com problemas de adição às drogas, organizados em grupos religiosos com o propósito de reabilitação(11). Os resultados mostraram que $75 \%$ dos jovens entrevistados começaram fumando tabaco, convidados por amigos das escolas, em sua iniciação. Procediam de famílias disfuncionais e permaneciam nessa situação porque não encontravam apoio na família que os rechaçava, sobretudo quando tomava conhecimento de que eles consumiam drogas.

Ao investigar as causas do consumo de drogas em um bairro de León, Nicarágua, outro estudo obteve resultados muito similares ${ }^{(12)}$. Uma das principais causas para o ingresso no consumo das drogas foi a falta de projeto de vida dos jovens para a transição para a vida adulta, assim como o fracasso escolar. Outro dado interessante apontado foi que os adolescentes entrevistados mencionaram a ausência de lugares de recreação e de oportunidades de trabalho, como algumas das motivações que induziram ao consumo de drogas. Finalmente, referiram a incompreensão dos pais ou tutores que, quase sempre, quando eles buscaram contato, alegaram não ter tempo para atendê-los em suas necessidades. Esses achados são convergentes aos obtidos por outras investigações, realizadas em contextos latino-americanos ${ }^{(13-15)}$.

A família é o núcleo básico da sociedade e, como todo grupo social, se encontra atravessada por papéis e posições que demarcam o comportamento do indivíduo. Cada membro exerce diferentes papéis na família que vão se alterando de acordo com a etapa do ciclo de vida de cada indivíduo. Os papéis assumidos podem ser agrupados em algumas categorias, que se modificam sistematicamente no decurso da vida: os papéis laborais, degênero e relativos à idade. As mudanças mais facilmente visualizadas incidem nos papéis familiares. Quando o adolescente começa a vivenciar novas experiências, nas quais deve assumir compromissos pessoais, familiares e sociais, e desempenha, ao mesmo tempo, dois ou mais papéis incompatíveis entre si, conflitos são gerados e a tensão resultante se manifesta quando não se tem habilidades para cumprir satisfatoriamente todos os papéis que the são adjudicados(16).

A busca pela identidade pessoal é um dos compromissos psicossociais mais decisivos para o adolescente, porquanto ele tem necessidade de se destacar em seu grupo familiar e de pares como ser distinto, dotado de personalidade própria. Nessa etapa do ciclo vital, ele busca dar sentido à vida e compreender aquilo que o cerca; para tanto, precisa aprender a aceitar a si mesmo e aos demais, fortalecendo seu ego. Para que esse processo de construção identitária se consolide, é importante que os pais estimulem o amadurecimento de seus filhos.

Esse período de intensas transformações biopsicossociais pode conduzir a um desenvolvimento 
saudável quando o núcleo familiar oferece uma boa base de sustentação para as experimentações do adolescente. Contudo, por vezes, a dinâmica familiar é conturbada e não contribui para acolher os conflitos dos filhos em desenvolvimento, o que pode levá-los a se engajar em comportamentos sintomáticos, que favorecem a aproximação ao universo das drogas.

Considerando esses pressupostos, este estudo teve por objetivo descrever características do contexto familiar de adolescentes de ensino secundário e explorar suas possíveis relações com o uso de substâncias psicoativas.

\section{Método}

Utilizou-se um desenho de estudo descritivo transversal, do tipo survey.

\section{Amostra}

Adolescentes de ambos os sexos, com idades entre 14 e 19 anos, que compreendem a adolescência média e tardia, segundo a definição da Organização Pan-Americana de Saúde. De um universo de 2.123 estudantes, participaram da pesquisa 657 adolescentes que constituíram a amostra final do estudo.

\section{Instrumento}

A coleta de dados foi realizada mediante questionário anônimo e autoadministrado. $\mathrm{O}$ instrumento foi elaborado tomando-se como referência - questionário da Organização Pan-Americana de Saúde, selecionando-se as perguntas que satisfaziam os objetivos do estudo.

Foi realizada avaliação prévia do questionário por meio de um estudo piloto, com 15 estudantes de um colégio com características semelhantes àqueles que seriam utilizados como cenários da investigação. Após a aplicação do instrumento, procedeu-se às correções e adaptações necessárias de algumas questões, que se revelaram de difícil compreensão pelos adolescentes.

\section{Procedimento}

$\mathrm{Na}$ planificação da investigação, consideraramse os adolescentes que seriam incluídos, formando o grupo, assim como os dois maiores colégios de educação secundária da cidade de Leon, Nicarágua. Esses colégios concentram a maior quantidade de adolescentes com idade entre 14 e 19 anos: o Instituto Nacional de Occidente (INO) e o Instituto Modesto Armijo Lozano (IMAL). No primeiro, do total de 1.021 estudantes do turno vespertino, participaram 311 estudantes entre $15 \mathrm{e}$ 19 anos. No segundo, de 1.102 estudantes, matriculados no turno vespertino, participaram 346 estudantes entre 15 e 19 anos.

Realizou-se, preliminarmente, aproximação com as autoridades do Ministério de Educação, explicandose as características do estudo, assim como seu potencial alcance para elaboração futura de estratégias de prevenção ao uso de substâncias psicoativas, nos diferentes colégios de educação secundária.

Após a avaliação e adaptação do questionário, o pesquisador obteve permissão das autoridades departamentais do Ministério da Educação para se inserir nos colégios; posteriormente, contou-se com a autorização dos diretores dos colégios e, finalmente, dos pais ou responsáveis legais pelos adolescentes com idade inferior a 18 anos que participaram da investigação. De modo pormenorizado, explicou-se a natureza científica do estudo, seus objetivos, bem como o caráter voluntário e anônimo da participação. Foram garantidos os direitos ao anonimato e à confidência das respostas.

Procedeu-se à aplicação dos questionários nas salas selecionadas. A coleta ocorreu em horários e locais que foram previamente acordados com a direção dos colégios. Aos estudantes que aceitaram participar era explicado, mais detalhadamente, o questionário e o modo de preenchimento. Reunidos em uma sala previamente preparada e sob condições de privacidade e conforto, o questionário era entregue. Uma vez concluído o preenchimento dos formulários, os adolescentes os depositavam em uma urna lacrada, na qual nada se via de seu conteúdo, garantindo-se, assim, a privacidade e anonimato do levantamento.

Depois de recolhidos os dados, procedeu-se à análise das informações coletadas. Posteriormente, as variáveis foram operacionalizadas e os dados tabulados e processados com auxílio do programa estatístico EpiInfo, versão 3.5.1.

O projeto foi aprovado pelo Comitê de Ética da Universidad Nacional Autónoma de Nicarágua, obedecendo a todos os requisitos para a condução de pesquisas envolvendo seres humanos.

\section{Resultados}

A Tabela 1 mostra que os estudantes que participaram do estudo eram, predominantemente, do sexo masculino, solteiros, católicos, tinham entre 15 e 16 anos, estavam cursando o quarto e quinto ano acadêmico e não exerciam atividade remunerada. 
Tabela 1 - Perfil dos adolescentes do ensino médio, participantes do estudo. León, Nicarágua, 2008

\begin{tabular}{|c|c|c|}
\hline Variáveis & $\mathbf{N}$ & $\%$ \\
\hline \multicolumn{3}{|l|}{ Sexo } \\
\hline Masculino & 347 & 52,8 \\
\hline Feminino & 310 & 47,2 \\
\hline Total & 657 & 100 \\
\hline \multicolumn{3}{|l|}{ Idade } \\
\hline 15 & 190 & 28,9 \\
\hline 16 & 200 & 30,4 \\
\hline 17 & 165 & 25,1 \\
\hline 18 & 69 & 10,5 \\
\hline 19 & 33 & 5,1 \\
\hline Total & 657 & 100 \\
\hline \multicolumn{3}{|l|}{ Estado civil } \\
\hline Solteiro & 623 & 94,9 \\
\hline Casado & 26 & 3,9 \\
\hline União consensual & 8 & 1,2 \\
\hline Total & 657 & 100 \\
\hline \multicolumn{3}{|l|}{ Ano acadêmico } \\
\hline I ano & 30 & 4,6 \\
\hline II ano & 61 & 9,3 \\
\hline III ano & 137 & 20,8 \\
\hline IV ano & 246 & 37,5 \\
\hline$V$ ano & 183 & 27,8 \\
\hline Total & 657 & 100 \\
\hline \multicolumn{3}{|l|}{ Religião } \\
\hline Católica & 300 & 45,7 \\
\hline Evangélica & 156 & 23,7 \\
\hline Nenhuma & 157 & 23,9 \\
\hline Outra & 44 & 6,7 \\
\hline Total & 657 & 100 \\
\hline \multicolumn{3}{|l|}{ Trabalho } \\
\hline Sim & 72 & 10,9 \\
\hline Não & 585 & 89,1 \\
\hline Total & 657 & 100 \\
\hline
\end{tabular}

A Tabela 2 mostra que, em relação às características do núcleo familiar, predominou o arranjo familiar intacto (convívio com ambos os pais - 56,1\%), seguido pelos adolescentes que viviam apenas com a mãe (31,6\%). O relacionamento com a mãe foi descrito como muito bom pela maioria dos adolescentes. No que concerne à relação de confiança com a mãe, predominou a categoria "muita", contudo, em porcentagem inferior à obtida em relação ao bom relacionamento com a mesma.

O relacionamento com o pai foi percebido como muito bom, porém, em porcentagem inferior ao relacionamento com a mãe. No que diz respeito à relação de confiança com o pai, predominou a categoria "pouca". Assim, pode-se dizer que, embora afirmem manter boas relações com a mãe e o pai, a confiança que têm neles revela diferenças substanciais, principalmente em relação à figura paterna que aparece como pouco confiável.

Tabela 2 - Características do núcleo familiar dos adolescentes estudantes do ensino médio. León, Nicarágua, 2008

\begin{tabular}{|c|c|c|}
\hline Variáveis & $\mathbf{N}$ & $\%$ \\
\hline \multicolumn{3}{|l|}{ Com quem vive em sua casa } \\
\hline Ambos os pais & 368 & 56,1 \\
\hline Só com a mãe & 208 & 31,6 \\
\hline Só com o pai & 16 & 2,4 \\
\hline Com nenhum dos dois & 8 & 1,2 \\
\hline Outros & 57 & 8,7 \\
\hline Total & 657 & 100 \\
\hline \multicolumn{3}{|c|}{ Relação de confiança com a mãe } \\
\hline Muita & 274 & 47,7 \\
\hline Moderada & 160 & 27,9 \\
\hline Pouca & 112 & 19,5 \\
\hline Nada & 28 & 4,9 \\
\hline Total & 574 & 100 \\
\hline \multicolumn{3}{|l|}{ Relacionamento com a mãe } \\
\hline Muito bom & 301 & 52,4 \\
\hline Bom & 190 & 33,1 \\
\hline Regular & 74 & 12,9 \\
\hline Mau & 9 & 1,6 \\
\hline Total & 574 & 100 \\
\hline \multicolumn{3}{|l|}{ Relação de confiança com o pai } \\
\hline Muita & 108 & 25,3 \\
\hline Moderada & 116 & 27,1 \\
\hline Pouca & 131 & 30,7 \\
\hline Nada & 72 & 16,8 \\
\hline Total & 427 & 100 \\
\hline \multicolumn{3}{|l|}{ Relacionamento com o pai } \\
\hline Muito bom & 158 & 37,0 \\
\hline Bom & 141 & 33,0 \\
\hline Regular & 92 & 21,6 \\
\hline Mau & 36 & 8,4 \\
\hline Total & 427 & 100 \\
\hline
\end{tabular}

Na Tabela 3, pode-se observar que adolescentes de ambos os sexos referiram uso regular de tabaco $(6,7 \%)$, cerveja $(5,2 \%)$ e licor $(n=4,7 \%)$, nessa ordem de frequência. Em relação às porcentagens de uso, os homens superaram em muito as mulheres no consumo de cigarro, cerveja e licor. Também chama a atenção o uso de drogas consideradas "mais pesadas", como cocaína, cola ("pega") e anfetamina. 
Tabela 3 - Frequência de uso e tipo de substâncias psicoativas, utilizadas pelos adolescentes do ensino médio, segundo o sexo. León, Nicarágua, 2008

\begin{tabular}{|c|c|c|c|c|}
\hline Drogas & Masculino & Feminino & Total & $\%$ \\
\hline \multicolumn{5}{|l|}{ Tabaco } \\
\hline Alguma vez & 135 & 48 & 183 & 27,8 \\
\hline Regularmente & $24=54,5 \%$ & $20=45,5 \%$ & 44 & 6,7 \\
\hline Total & 159 & 68 & 227 & 34,5 \\
\hline \multicolumn{5}{|l|}{ Cerveja } \\
\hline Alguma vez & 121 & 50 & 171 & 26,0 \\
\hline Regularmente & $19=55,9 \%$ & $15=44,1 \%$ & 34 & 5,2 \\
\hline Total & 140 & 65 & 205 & 31,2 \\
\hline \multicolumn{5}{|l|}{ Licor } \\
\hline Alguma vez & 92 & 37 & 129 & 19,6 \\
\hline Regularmente & $19=61,3 \%$ & $12=38,7 \%$ & 31 & 4,7 \\
\hline Total & 111 & 49 & 160 & 24,3 \\
\hline \multicolumn{5}{|l|}{ Cocaína } \\
\hline Alguma vez & 23 & 7 & 30 & 4,5 \\
\hline Regularmente & - & - & - & - \\
\hline Total & 23 & 7 & 30 & 4,5 \\
\hline \multicolumn{5}{|l|}{ Anfetaminas } \\
\hline Alguma vez & 17 & 6 & 23 & 3,5 \\
\hline Regularmente & 1 & - & 1 & 0,1 \\
\hline Total & 18 & 6 & 24 & 3,6 \\
\hline \multicolumn{5}{|l|}{ Cola } \\
\hline Alguma vez & 21 & 7 & 28 & 4,3 \\
\hline Regularmente & 1 & - & 1 & 0,1 \\
\hline Total & 22 & 7 & 29 & 4,4 \\
\hline
\end{tabular}

A Tabela 4 mostra que a idade de início do uso de substâncias concentra-se, principalmente, nos 12 anos, seguidos dos 13 anos. Dos 227 que iniciaram uso de tabaco, 70 o fizeram aos 12 anos e 68 aos 13 anos, havendo distribuição numérica similar para o uso de cerveja e licor.

Tabela 4 - Idade de início e tipo de substância psicoativa mais utilizada pelos adolescentes do ensino médio. León, Nicarágua, 2008

\begin{tabular}{|c|c|c|c|c|c|c|c|}
\hline Idade & Tabaco & Cerveja & Licor & Cocaína & Anfetamina & Cola & Total \\
\hline 11 & 13 & 12 & 7 & 2 & 2 & 4 & 40 \\
\hline 12 & 70 & 60 & 54 & 10 & 10 & 8 & 212 \\
\hline 13 & 68 & 62 & 46 & 12 & 8 & 10 & 196 \\
\hline 14 & 52 & 47 & 40 & 4 & 3 & 5 & 151 \\
\hline 15 & 21 & 23 & 12 & 2 & 1 & 1 & 60 \\
\hline 16 & 2 & 1 & 1 & - & - & 1 & 5 \\
\hline 17 & 1 & - & - & - & - & - & 1 \\
\hline Total & 227 & 205 & 160 & 30 & 24 & 29 & - \\
\hline
\end{tabular}

Questionados de que maneira se iniciaram no uso das drogas, os adolescentes declararam: por amigos $(49,0 \%)$, por conta própria $(36,1 \%)$, por influência de um familiar $(14,8 \%)$. As motivações alegadas para o uso foram: curiosidade $(80,6 \%)$, desgosto com os pais $(8,3 \%)$, pressão de amigos $(5,7 \%)$ e obrigado por amigos $(5,3 \%)$.
Com relação ao uso de substâncias psicoativas na família, $340(51,7 \%)$ adolescentes responderam afirmativamente e 317 (48,3\%) negaram o uso. As respostas à questão: "quem, na família, faz uso dessas substâncias?" apontaram: outros (165-48,5\%), pai (143-42,1\%), irmão (24-7\%) e mãe (8-2,4\%). 
Ao verificar a relação entre uso de substâncias e a estrutura do núcleo familiar a que pertencem os adolescentes, constatou-se, de forma surpreendente, que a maior quantidade de usuários de substâncias psicoativas provém de lares nos quais está presente o casal parental, seguido por famílias matrifocais.

Em relação à exposição à violência intrafamiliar, observou-se que 203 adolescentes (30,9\%) vivenciavam, frequentemente, situações de rixas nos lares (30,9\%); 82 respondentes $(12,5 \%)$ haviam sido expostos à violência verbal, com frequência, nos últimos 12 meses; 356 (54,2\%) expressaram ter sofrido algumas vezes; nove $(1,4 \%)$ assinalaram que sofreram maustratos físicos, com frequência, nos últimos 12 meses; $14(2,1 \%)$ manifestaram ter sofrido abuso sexual. Essas porcentagens são preocupantes ao se considerar que adolescentes não deveriam sofrer nenhum tipo de violência intrafamiliar, caracterizando exposição à situação de vulnerabilidade.

\section{Discussão}

Revisando os resultados obtidos, percebe-se que a amostra apresentou porcentagens similares quanto ao sexo. Em relação à idade, a faixa etária é compatível aos anos acadêmicos que estão cursando - entre o IV e o V ano da escola secundária. Como era de se esperar, a maioria dos adolescentes era solteira, semelhante aos dados obtidos por outros estudos.

Por outro lado, observa-se que, em relação às características do núcleo familiar de proveniência, pouco mais da metade dos adolescentes provém de lares intactos, nos quais ambos os progenitores estão presentes. Contudo, a relação de confiança, sobretudo com relação ao pai, é bastante limitada, o que impõe restrições à comunicação e expressão de sentimentos e necessidades afetivas por parte do adolescente.

O consumo de drogas, em relação ao sexo, revela que os adolescentes superam em quase $50 \%$ o uso por parte das mulheres nas três substâncias majoritariamente utilizadas nessa amostra: tabaco, cerveja e licor. Os dados obtidos acerca do início do uso de substâncias pelos adolescentes - 12 anos, seguidos dos 13 e 14 anos - foram cruzados com o total de participantes que fizeram uso de tabaco (121), cerveja (196) e licor (151). Esses dados são compatíveis com os resultados obtidos por estudo realizado na cidade do México $^{(7)}$.

Os achados sobre os motivos que impulsionaram os adolescentes a utilizar drogas não se diferenciam dos estudos anteriores, bem como a questão de por quem, frequentemente, se veem pressionados para seu consumo(7-8). A curiosidade para experimentar novas sensações é o elemento principal que os impulsionou a se aproximarem das drogas, assim como a pressão dos amigos. Por outro lado, 52\% das famílias de procedência dos adolescentes têm antecedentes de uso de drogas, o que corresponde, em números absolutos, a 340 famílias. A figura paterna é, de longe, a que mais faz uso de substâncias (43\%).

Constatou-se, finalmente, nas famílias desses adolescentes, a presença de discussões, incidência de maus-tratos verbais e físicos, bem como abuso sexual, o que indica que a violência intrafamiliar pode ser fator de risco para o uso de substâncias pelos adolescentes deste estudo, sobretudo quando combinada com o uso de tabaco e cerveja. Esses achados corroboram evidências da literatura, indicando que os adolescentes que consomem drogas ilícitas enfrentam problemas familiares $^{(17)}$.

\section{Conclusões}

A presente investigação foi desenvolvida em dois colégios de ensino fundamental da cidade de Leon, Nicarágua. O interesse neste trabalho repousa na necessidade de se reconhecer as características do núcleo familiar de procedência de adolescentes de 14 a 19 anos de idade, que admitiram haver consumido substâncias psicoativas.

O total de adolescentes participantes do estudo foi de 657 , dos quais 347 eram homens. As idades nas quais se situava o maior número de adolescentes foi de 16 (31\%) e 15 (29\%) anos, seguidos pelos participantes de 17 (25\%) anos; 95\% da amostra era composta por solteiros; a maior parte cursava o IV e $\mathrm{V}$ ano da escola secundária; $46 \%$ se referiu como católico e $90 \%$ não trabalhava; $56 \%$ deles conviviam com ambos os genitores e $32 \%$ somente com a mãe; $86 \%$ admitiu ter boas relações com a mãe, mas não tanta confiança (24\%).

Os adolescentes que usam substâncias eram procedentes de famílias intactas, seguidas por famílias nas quais somente a figura materna estava presente. Quanto aos tipos de droga mais utilizados, o levantamento resultou nessa ordem: tabaco, cerveja e licor. Os rapazes superaram as mulheres no uso dessas substâncias. A idade de início do uso de substâncias foi 12 anos, seguida pelos adolescentes de 13 e 14 anos, sendo as razões alegadas para o consumo a curiosidade para experimentar novas sensações e a pressão de amigos. 
Em relação ao uso de substâncias no núcleo familiar, 52\% das famílias, das quais os adolescentes procedem, apresentavam antecedentes de uso, sendo o pai o membro que normalmente utiliza (42\%), principalmente licor e tabaco. Finalmente, observouse que os adolescentes que usam alguma substância, especialmente tabaco, cerveja e licor, procedem de famílias intactas, seguidas por famílias nas quais apenas a figura materna está presente. Ou seja, o maior número de adolescentes que utiliza drogas provinha de núcleos familiares nos quais ambos os pais estavam presentes. A presença de violência verbal e física, inclusive sexual, também foram ocorrências relacionadas ao uso de drogas, fundamentalmente lícitas, pelos adolescentes deste estudo.

Contribuir para redução do consumo das drogas entre adolescentes deve ser tarefa prioritária de todos os profissionais de saúde, principalmente os educadores, que estão formando os futuros profissionais de saúde na Nicarágua. Nesse sentido, é preciso que as autoridades educacionais e sanitárias interajam com trabalho em rede, desenvolvendo ações para prevenção ao uso de substâncias em idade precoce. Além disso, é necessário reforçar a vigilância para exigir o cumprimento da lei, em relação à proibição da venda de licor e cigarro a menores de 18 anos. Campanhas devem ser planejadas a fim de informar sobre as consequências deletérias do tabaco tanto para a saúde como para o entorno familiar e comunitário.

Por meio dos conselhos de classe, os colégios devem investir em atividades que incidam sobre o comportamento dos pais de família que utilizam regularmentealguma substância psicoativa, considerando que eles funcionam como modelos de conduta para seus filhos. Jovens e adolescentes encontram-se em delicada fase de transição psicossocial, na qual buscam consolidar sua identidade, por meio dos processos identificatórios. No decorrer desse processo, o meio familiar é um dos contextos privilegiados para a busca de modelos de comportamento com os quais se possa identificar. Os adultos, mediante seu funcionamento psicológico e seus modos de resolução de conflitos, oferecem exemplos a serem ou não seguidos pelos adolescentes.

São necessários, finalmente, investimentos para desenvolver locais de socialização e recreação saudáveis, para que os adolescentes possam canalizar as energias próprias da juventude, no sentido de atividades socialmente úteis e relevantes. Essas ações podem funcionar como fatores protetores contra o uso de substâncias na adolescência. Para os adolescentes que se encontram em situação de vulnerabilidade psicossocial, recomenda-se a disponibilidade de dispositivos de assistência compatíveis com as necessidades da juventude. Os dados apresentados fornecem subsídios e insumos para a reorganização das práticas em saúde, com vistas a aprimorar a atenção à saúde dos adolescentes.

\section{Agradecimentos}

Agradecemos a Comissão Interamericana para o Controle do Abuso de Drogas/CICAD da Secretaria de Segurança Multidimensional/SSM da Organização dos Estados Americanos/OEA, a Secretaria Nacional de Políticas sobre Drogas/SENAD do Gabinete de Segurança Institucional/Brasil, a Escola de Enfermagem de Ribeirão Preto da Universidade de São Paulo e o Centro Colaborador da Organização Mundial da Saúde para o Desenvolvimento da Pesquisa em Enfermagem, a população representada nas pesquisas, bem como as autoridades das universidades representadas pelos participantes do Programa On-Line de Especialização em Pesquisa sobre o Fenômeno das Drogas, períodos 2006, 2007, 2008 e 2009.

\section{Referências}

1. Pratta EMM, Santos MA. O processo saúde-doença e a dependência química: interfaces e evolução. Psicol Teoria e Pesqui. 2009;25(2):203-11.

2. Silber TJ, Munist MM, Maddaleno M, Suárez Ojeda EN, organizadores. Manual de medicina de la adolescencia. Washington DC: OPS; 1992.

3. Rebolledo EA, Medina NMO, Pillon SC. Factores de riesgo asociados al uso de drogas em estudiantes adolescentes. Rev. Latino-Am. Enfermagem. 2004;12(no especial):369-75.

4. Pratta EMM, Santos MA. Adolescence and the consumption of psychoactive substances: the impact of the socioeconomic status. Rev. Latino-Am. Enfermagem. 2007;15(número especial):806-11.

5. Martins M, Santos MA, Pillon SC. Low-income families' perceptions on the use of drugs by one of their members. Rev. Latino-Am. Enfermagem. 2008;16(2):293-8.

6. García J. Ministerio de Salud de la República de Nicaragua. Manual de atención y consejería para adolescentes. UNICEF: Editorial Universitaria; 1998.

7. Jinez MLJ, Souza JRM, Pillon SC. Uso de drogas y factores de riesgo entre estudiantes de enseñanza media. Rev. Latino-Am. Enfermagem. 2009;17(2):24652. 
8. Pratta EMM, Santos MA. Levantamento dos motivos e dos responsáveis pelo primeiro contato de adolescentes do ensino médio com substâncias psicoativas. SMAD 2006;2(2). [acesso em: 03 dezembro 2009]. Disponível em: http://www2.eerp.usp.br/resmad/

9. Pratta EMM, Santos MA. Família e adolescência: a influência do contexto familiar no desenvolvimento psicológico de seus membros. Psicol Estudo (Maringá) 2007;12(2):247-56.

10. Ministerio de Salud de la República de Nicaragua. Dirección de Atención Integral a la Mujer, Niñez y Adolescencia. MINSA/UNFPA. Managua, Nicaragua: Editorial Somarraba; 1999.

11. Ulloa W, Ugarte W, Rodríguez R. Abandonar la drogadicción: algo mas que simplemente decir no a las drogas. León - Nicaragua; 2000.

12. Munguía D, Pacheco G, Pallais F. Causas del consumo de las drogas en los adolescentes del barrio de Sitiaba. León - Nicaragua; 2001.

13. Pratta EMM, Santos MA. Opiniões dos adolescentes do ensino médio sobre o relacionamento familiar e seus planos para o futuro. Paideia (Ribeirão Preto) 2007;17(36):103-14.

14. Pratta EMM, Santos MA. Lazer e uso de substâncias psicoativas na adolescência: possíveis relações. Psicol Teoria e Pesqui 2007;23(1):43-52.

15. Pratta EMM, Santos MA. Reflexões sobre as relações entre drogadição, adolescência e família: um estudo bibliográfico. Estudos Psicol (Natal) 2006;11(3):31522.

16. Bee H. A criança em desenvolvimento. Porto Alegre: Artmed; 1996.

17. Martinez LC, Ferriani MGC. Relación entre las características de la adolescente embarazada y la resistencia al consumo de droga. Rev. Latino-Am. Enfermagem. 2004;12(no.spe):333-9. 